\title{
METHOD FOR THE DETECTION OF MIXED QPSK SigNALS BASED ON THE CALCULATION OF FOURTH-ORDER CUMULANTS
}

\author{
Vasyl Semenov, Pavel Omelchenko and Oleh Kruhlyk \\ Department of Algorithms, Delta SPE LLC, Kiev, Ukraine
}

\begin{abstract}
In this paper we propose the method for the detection of Carrier-in-Carrier signals using QPSK modulations. The method is based on the calculation of fourth-order cumulants. In accordance with the methodology based on the Receiver Operating Characteristic (ROC) curve, a threshold value for the decision rule is established. It was found that the proposed method provides the correct detection of the sum of QPSK signals for a wide range of signal-to-noise ratios and also for the different bandwidths of mixed signals. The obtained results indicate the high efficiency of the proposed detection method. The advantage of the proposed detection method over the "radiuses" method is also shown.
\end{abstract}

\section{KEYWORDS}

Carrier-in-Carrier, Cumulants, QPSK, Receiver Operating Curve.

\section{INTRODUCTION}

The development of digital communications creates the need to develop more efficient methods for using frequency resources. Although modern methods of error-correction coding allow working at rates close to the theoretical Shannon limit, channel capacity can be increased by using Carrier-in-Carrier (also known as PCMA, Paired-Carrier Multiple Access) technologies in which multiple transmitted signals occupy the same band (see, for example, [1]).

The Carrier-in-Carrier modems usually operate in the same bandwidth using well-known echo compensation techniques. However, in such applications as non-cooperative communications, radio reconnaissance tasks and spectrum analysis there is a need to:

A. detect the presence of $\mathrm{CnC}$ signal;

B. perform the blind separation of two (or more) signals.

There are various methods of blind separation for the sum of several signals, including Bayesian estimation of signals [2-3], Particle Filtering [4], neural networks [], PCI (Principal informative components), ICA (Independent Components Analysis) etc. Although there is a variety of blind separation methods, the problem is still far from being solved.

At the same time, the task of detecting the fact of transmitting a superposition of signals at a given frequency is practically not addressed in the literature. Therefore, in this paper we propose a method for detecting the sum of two QPSK signals based on the use of the fourth-order cumulants. Cumulants, which are high-order mixed moments, are widely used in signal processing problems (see, for example, [6]). For example, there are many methods for applying them to the problem of detecting the modulation type of a transmitted signal (see [7]). It was shown that cumulants have high efficiency in detecting the type of signal modulation. This motivated our study of the application of cumulants to the problem of detecting Carrier-in-Carrier signals. 
This paper provides an algorithm for the classification of Carrier-in-Carrier signals using a fourthorder cumulants. To select the optimal decision threshold, we used the Area Under the Curve (AUC) criterion (see, for example, [8]). Numerical simulations confirm the effectiveness of the proposed method.

\section{Preliminaries}

Suppose we have the sum of two QPSK as the observational signal $x(t)$ :

$$
x(t)=x_{1}(t)+x_{2}(t)+w(t)
$$

Where $x_{u}(t), u=1,2$ are the signals from two sources:

$$
x_{u}(t)=a_{u} e^{j \phi_{u}} \sum_{n=-\infty}^{\infty} s_{u}(n) g\left(t-n T_{u}-\tau_{u}\right), u=1,2
$$

and $s_{u}(n), u=1,2$ are original sequences to be estimated; $T_{u}$ are the symbol periods; $a_{u}$ are the amplitudes; $\phi_{u}$ are the phases; $\tau_{u}$ are the time shifts; $g(t)$ is a total channel response (assumed to be raised square-root cosine with known roll-off); $w(t)$ is a white Gaussian noise. The principle of formation of the received signal is shown in Figure 1.

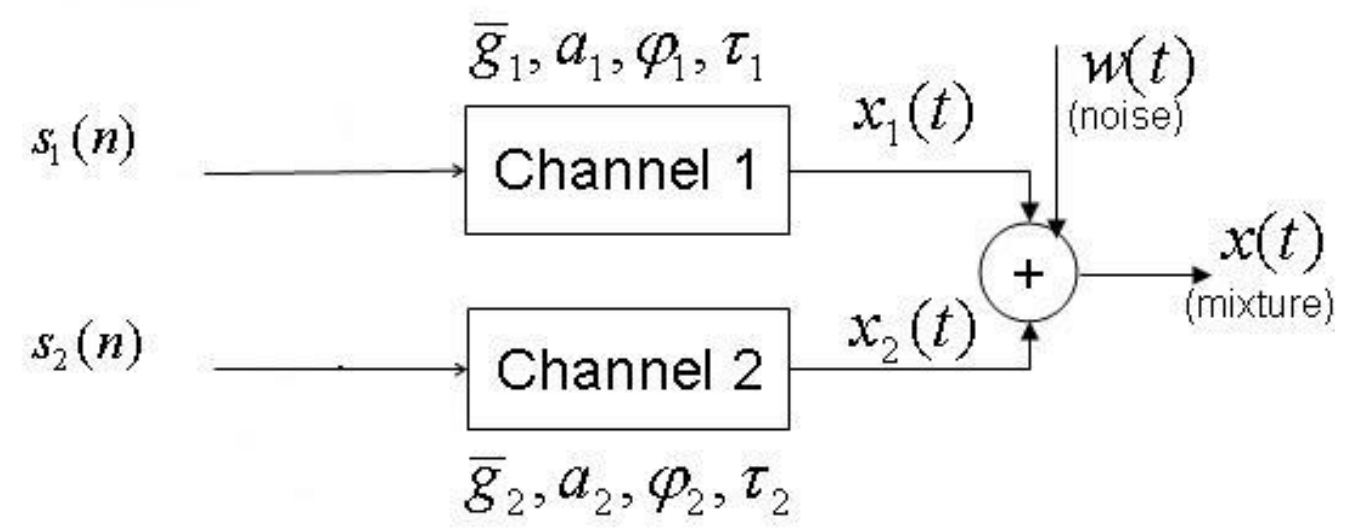

Figure 1. Statement of the problem.

The main criterion of the proposed method of detecting carrier-in-carrier in the channel is based on the demodulator's output constellation. Typical signal constellations of the QPSK (Quaternary Phase Shift Keying) signals in the presence of noise with SNR (signal-to-noise ratio) equal to 10 $\mathrm{dB}$ are shown in Figure 2 (a) (here $x_{2}(t)=0$ ).

On the other hand, the carrier-in-carrier constellation has a distribution of points along circles around nominal points for simple QPSK modulation (Figure 2 (b)). This kind of constellation arises because of the sum of two M-PSK signals with a small difference of the carrier frequencies. The radius of the circle of distribution of points around the nominal point of the constellation depends on the ratio of the amplitudes of the signals, i.e. the greater the ratio of the amplitudes $a_{2} / a_{1}$, the smaller is the radius of the circle (see Figure 3). 
Signal \& Image Processing: An International Journal (SIPIJ) Vol.10, No.3, June 2019

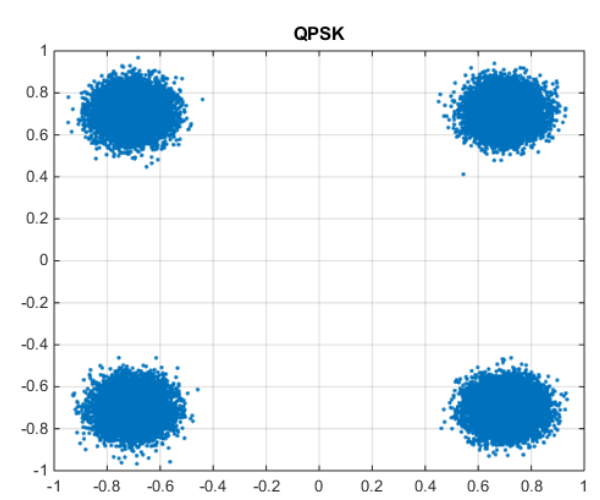

a

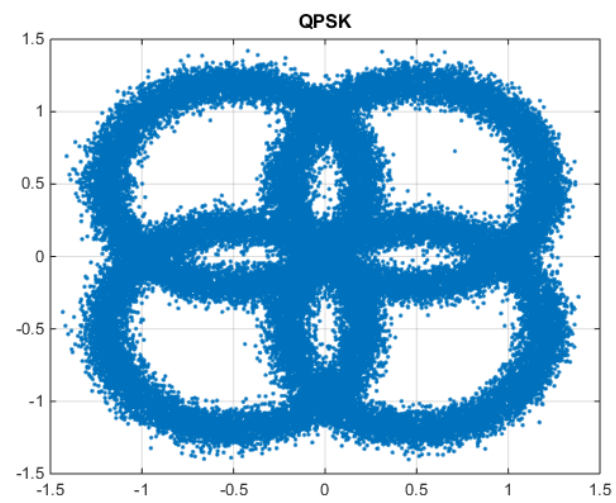

b

Figure 2. Output constellations for the normal QPSK signal (a) and the sum of two QPSK signals with equal amplitudes (b).

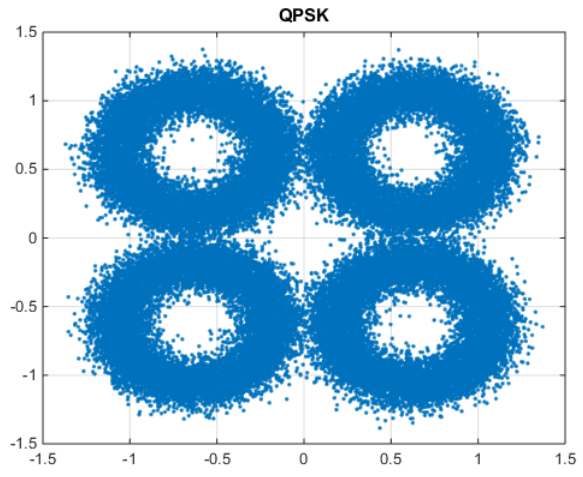

a

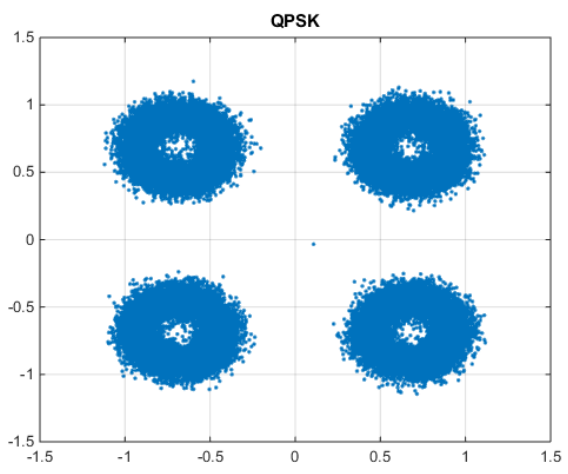

b

Figure 3. Output constellations for the sum of two QPSK signals with a ratio of amplitudes 2 (a) and 4 (b) respectively.

Therefore, our proposed method for detecting Carrier-in-Carrier signals is based on detecting the transformation of the signal constellation. In accordance with this method, a fourth-order joint cumulant is calculated between the signal and its complex conjugate copy in one quadrant of the complex constellation.

The proposed detection algorithm is as follows. Signal samples are selected that correspond to one of the quadrants in the complex constellation (Figure 4):

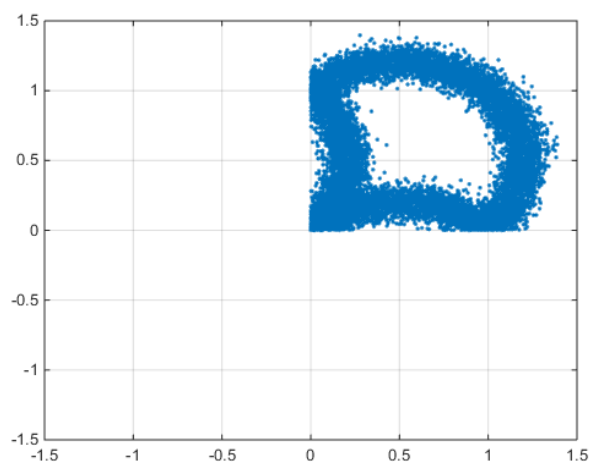

Figure 4. The QPSK samples corresponding to one of the quadrants of the complex constellation. 
Then the average value is subtracted from the received signal. Denote the received centered signal as $x(n)$. Then the value for the detection of Carrier-in-Carrier signals is fourth order cumulant:

$$
C_{2,2}=\operatorname{cum}\left[x, x, x^{*}, x^{*}\right]
$$

Where $x^{*}$ is the conjugated signal.

The proposed criterion for detecting Carrier-in-Carrier signals consists of comparing the cumulant (1) with a threshold value. In that case, if the value defined by formula (1) does not exceed the threshold:

$$
C_{2,2}<\Pi_{C}
$$

Then a decision is made about the presence of a Carrier-in-Carrier signal. Otherwise, a decision is made about the absence of a Carrier-in-Carrier signal. The choice of the threshold will be discussed in the next section.

As is known, the quantity (1) can be expressed in terms of statistical moments:

$$
C_{2,2}=E_{2,2}-\left|E_{2,0}\right|^{2}-2 E_{1,1}^{2},
$$

Where $E_{m, n}$ is the joint moment of the order $(m, n)$ :

$$
E_{m, n}=\frac{1}{N} \sum_{k=1}^{N} x^{m}(k)\left(x^{*}(k)\right)^{n}
$$

From here follow simple formulas for calculating of $E_{1,1}$ and $E_{2,0}$ :

$$
\begin{aligned}
& E_{2,0}=\frac{1}{N} \sum_{k=1}^{N} x^{2}(k), \\
& E_{1,1}=\frac{1}{N} \sum_{k=1}^{N}|x(k)|^{2}
\end{aligned}
$$

In addition, in this paper, we compare the proposed detection method with the approach based on the standard deviation of the average radius of the signal constellation points in one quadrant of the complex constellation. In accordance with this criterion, the standard deviation for the calculated distances $|x(n)|$ is calculated normalized to the square of their average value:

$$
\sigma_{r}=\frac{1}{e_{x}^{2}} \sqrt{\frac{1}{N} \sum_{k=1}^{N}\left(|x(n)|-e_{x}\right)^{2}},
$$

Where

$$
e_{x}=\frac{1}{N} \sum_{k=1}^{N}|x(n)|
$$


In accordance with this approach, in the event that the value $\sigma_{r}$ defined by formula (3) does not exceed the threshold $\Pi_{r}$ :

$$
\sigma_{r}<\Pi_{r}
$$

Then a decision is made about the presence of a Carrier-in-Carrier signal. Otherwise, a decision is made about the absence of a Carrier-in-Carrier signal.

\section{Additional Criteria and Full Structure of Detection Method}

As an alternative criterion for the detection of sum of QPSK signals, we propose to use the detection of presence of several carrier frequencies in the observed signal. In the case of QPSK it can be detected by the analysis of the spectrum of fourth power of observed signal. The example is given at the Figure 5.

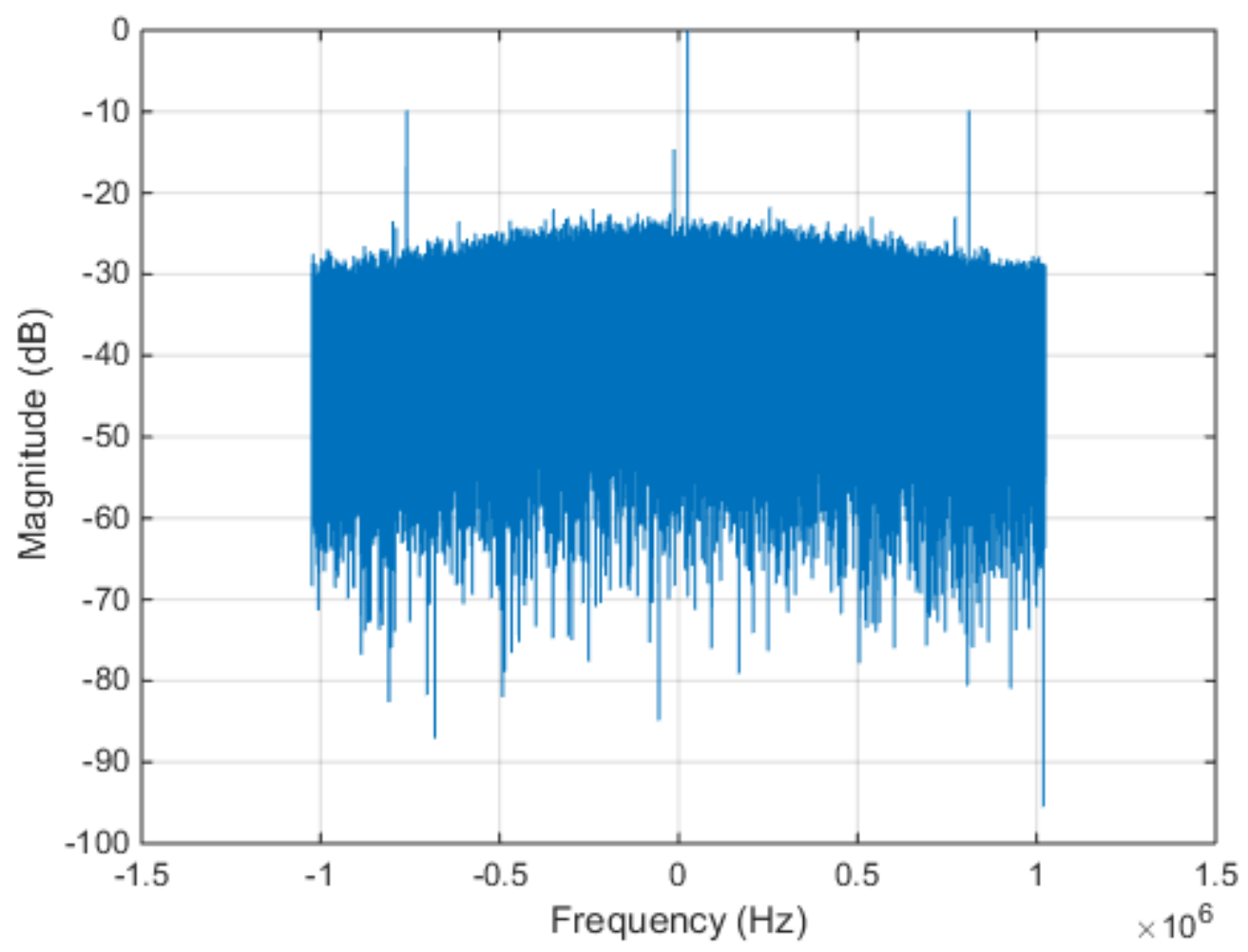

Figure 5. Power spectrum of the fourth power of Carrier-in-Carrier signal.

The presence of multiple peaks in this spectrum can be used as an auxiliary confirmation of the presence of multiple signals at the given frequency. Thus, the proposed criterion for the detection of Carrier-in-Carrier signals can be facilitated by the detection of multiple peaks in the power spectrum of the observed signal. 


\section{EXPERIMENTAL RESULTS}

In the following experiments, the cumulant values (1) and the normalized constellation radiuses (3) were calculated for signal-to-noise ratios (SNR) from 0 to $10 \mathrm{~dB}$ with an amplitude ratio of 0 (case of a single signal, $\left.x_{2}(t)=0\right), 1,2,4$ and also for the additive white Gaussian noise. Here we assume that the signals have the same symbol period, although we will comment below that the proposed approach can be applied to signals with different symbol periods.

For the modeling we use the following scheme of experiment presented at Figure 6. The results of calculating the parameter (1) are shown in Figure 7.

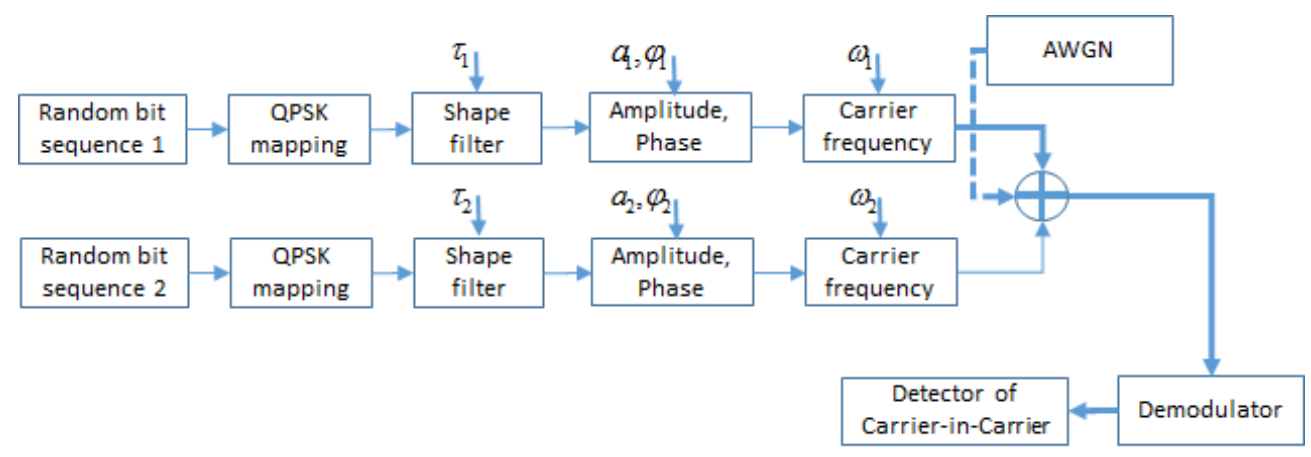

Figure 6. The scheme of modeling experiment.

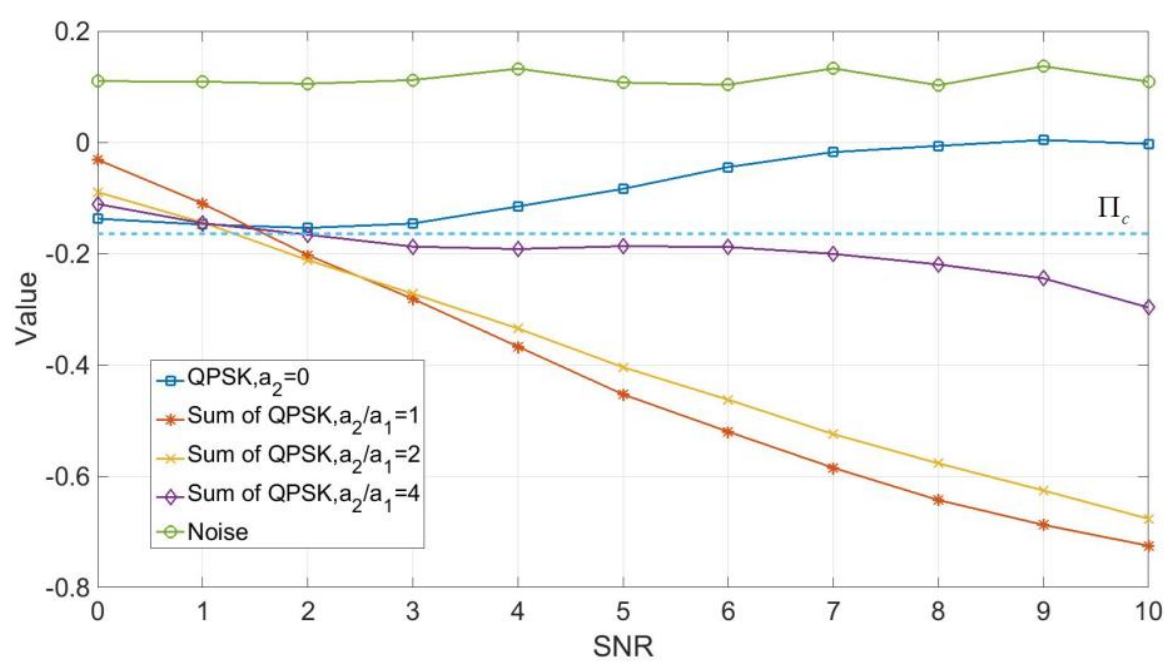

Figure 7. Dependence of average cumulants on the SNR for various ratios of the amplitudes of the signals.

Figure 7 shows that the cumulant plots corresponding to Carrier-in-Carrier are separated from the plots corresponding to a single QPSK signal and background noise in the case when SNR is greater than $1.5 \mathrm{~dB}$ (which is a common situation in practice). The "cumulants" method ensures the correct detection of a Carrier-in-Carrier (by comparing with a given threshold) for different ratios of signal amplitudes. It can also be seen that the cumulant graph (1), corresponding to white noise, is clearly separated from the cumulant graphs corresponding to the three considered cases of the sum of QPSK signals. 
The results of calculating the parameter (3) for the radius method are shown in Figure 8. As can be seen from the figure, the detector based on the constellation radius does not provide a clear separation of the radius values necessary for the correct detection.

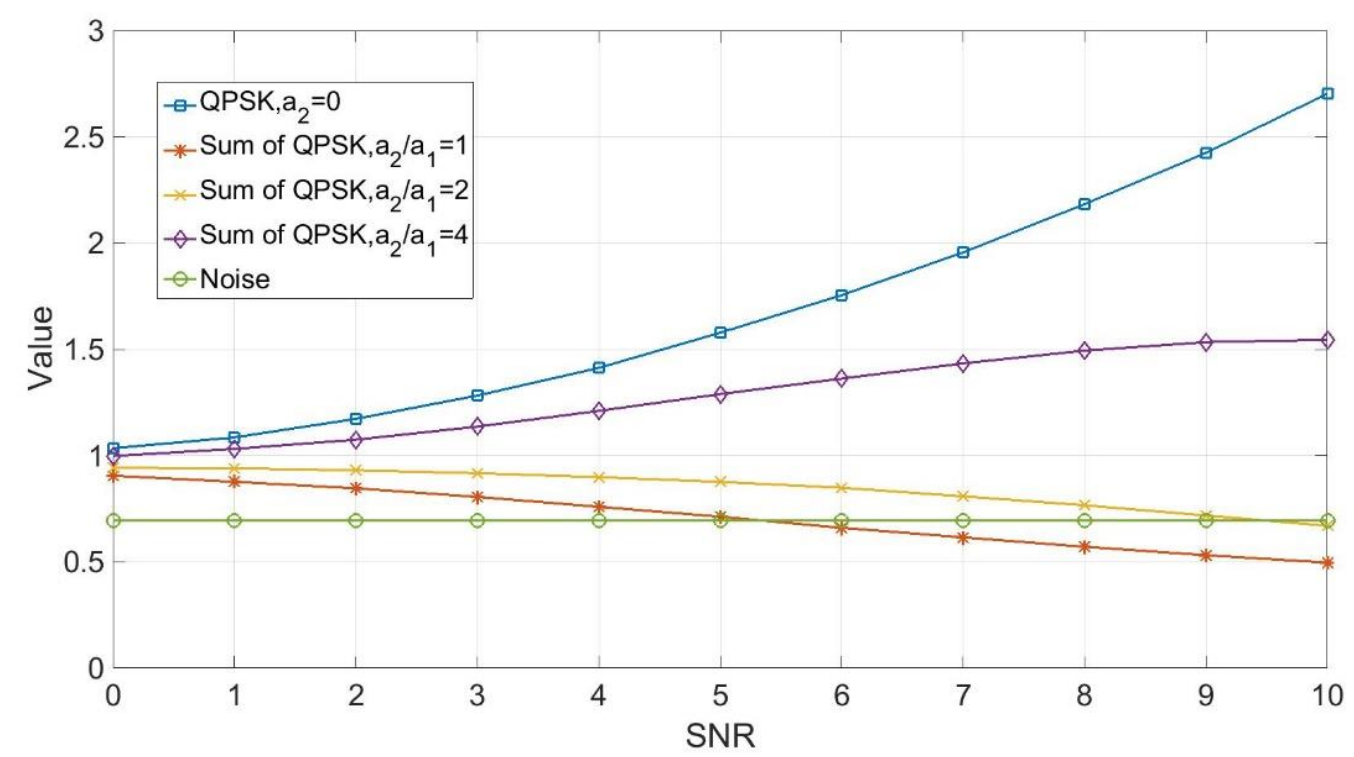

Figure 8. Dependence of normalized constellation radiuses on the SNR for various ratios of the amplitudes of the signals.

In the next step, it is necessary to select the optimal thresholds for the rules (2) and (4). To select these thresholds and test the overall effectiveness of the proposed rule for detecting the Carrierin-Carrier signals, we will use a technique based on the use of the ROC curve or, namely, Area under the Curve (AUC) parameter [8]. The calculation of this parameter requires the calculation of the statistical characteristics of a true positive rate (probability of correct detection) and a false positive rate (probability of false alarm) for all possible threshold values that lie within the range of values of the parameter selected as the detection criterion. The AUC plots for the cumulants method and the radius method are presented in Figures 9 and 10 respectively.

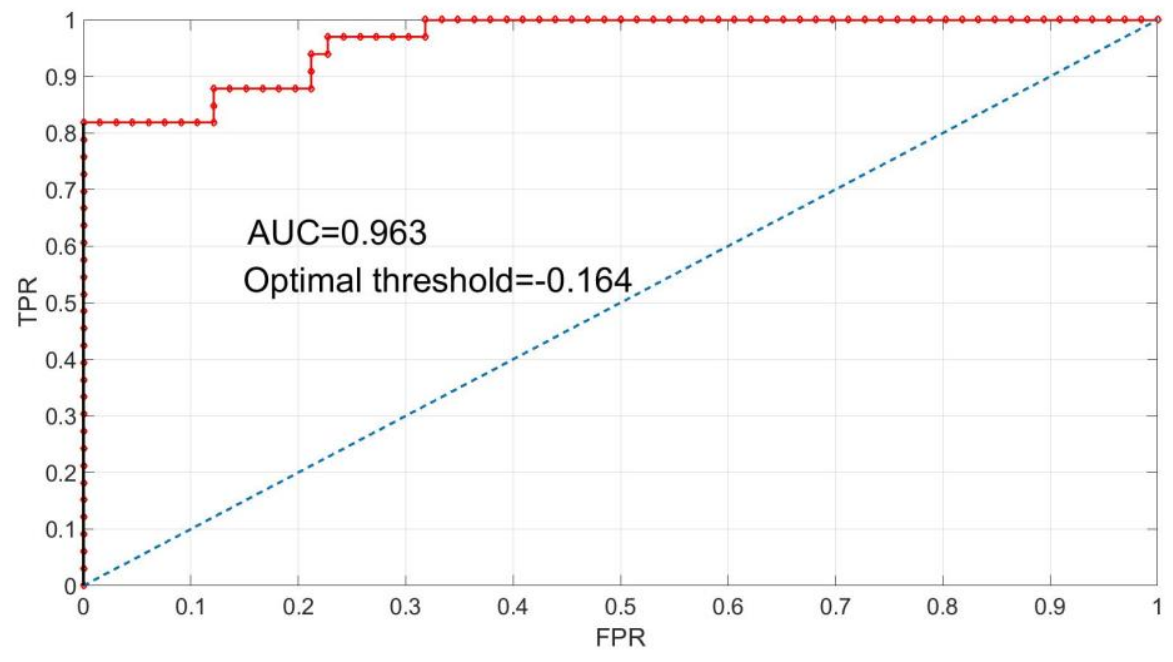

Figure 9: ROC curve for the cumulants method . 


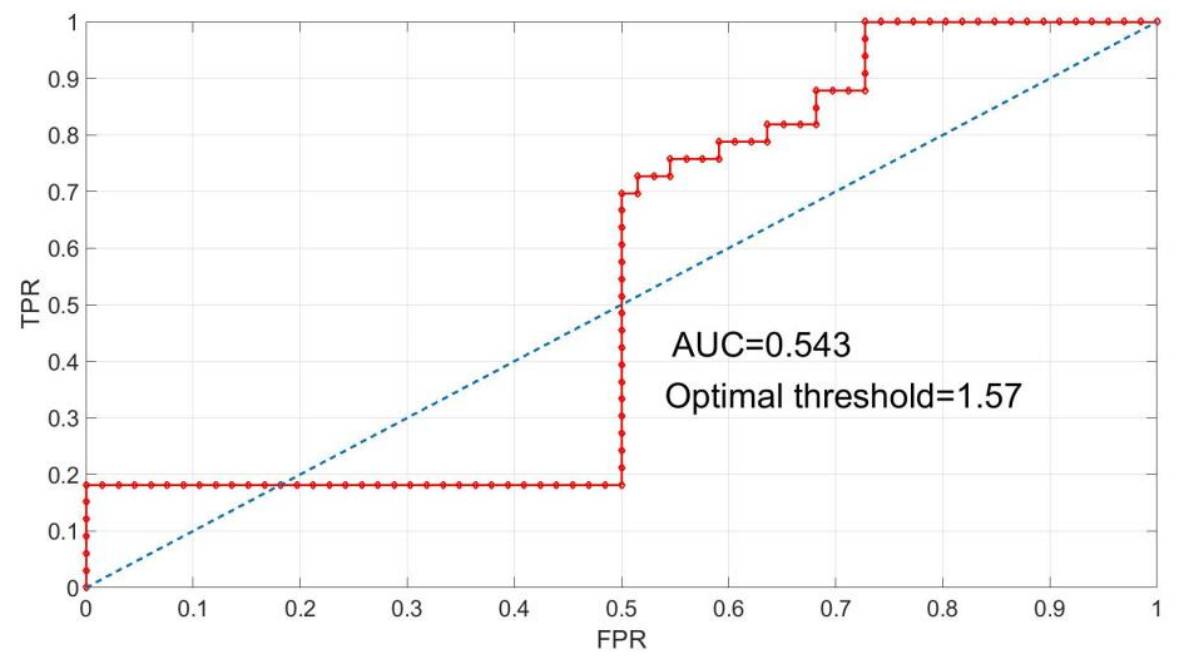

Figure 10. ROC curve for the radiuses method.

From Figure 9 it follows that the AUC parameter for the cumulants method is 0.963 , which indicates the high reliability of the proposed detection method. This is because the threshold $\Pi_{C}=-0.164$ chosen for the cumulants method is equally well suited for all SNRs and signal amplitude ratios. At the same time, the AUC value for the radiuses method (Figure 10) is significantly lower (0.543). The radiuses method is very sensitive to changes in the ratio of the amplitudes of the summable signals, as well as to variations of other parameters.

We also considered the implementation of proposed method to the detection of sum of QPSK signals with different symbol rates. The average cumulant values for the different SNRs are shown at the Figures 11-12 for the cases $T_{2} / T_{1}=0.25, a_{2} / a_{1}=1$ and $T_{2} / T_{1}=0.25, a_{2} / a_{1}=2$ respectively.

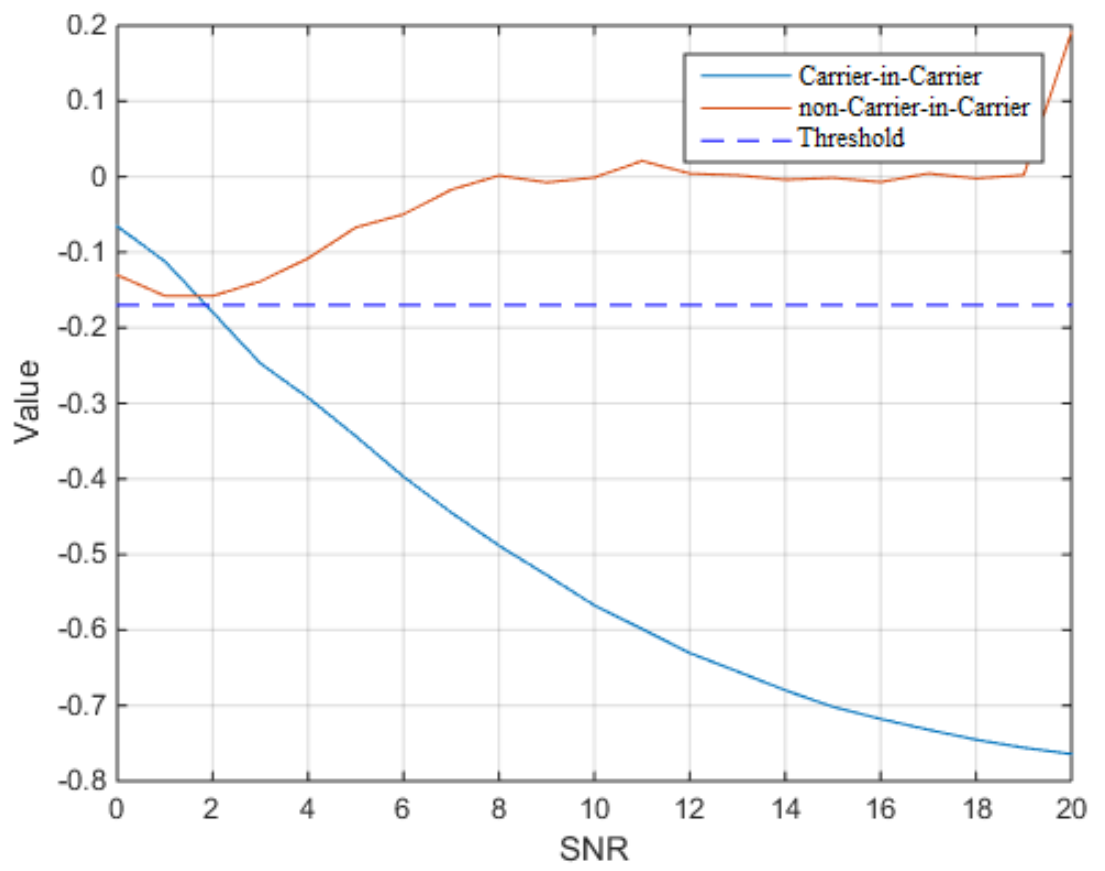

Figure 11. Dependence of average cumulants on the SNR for the case $T_{2} / T_{1}=0.25, a_{2} / a_{1}=1$. 


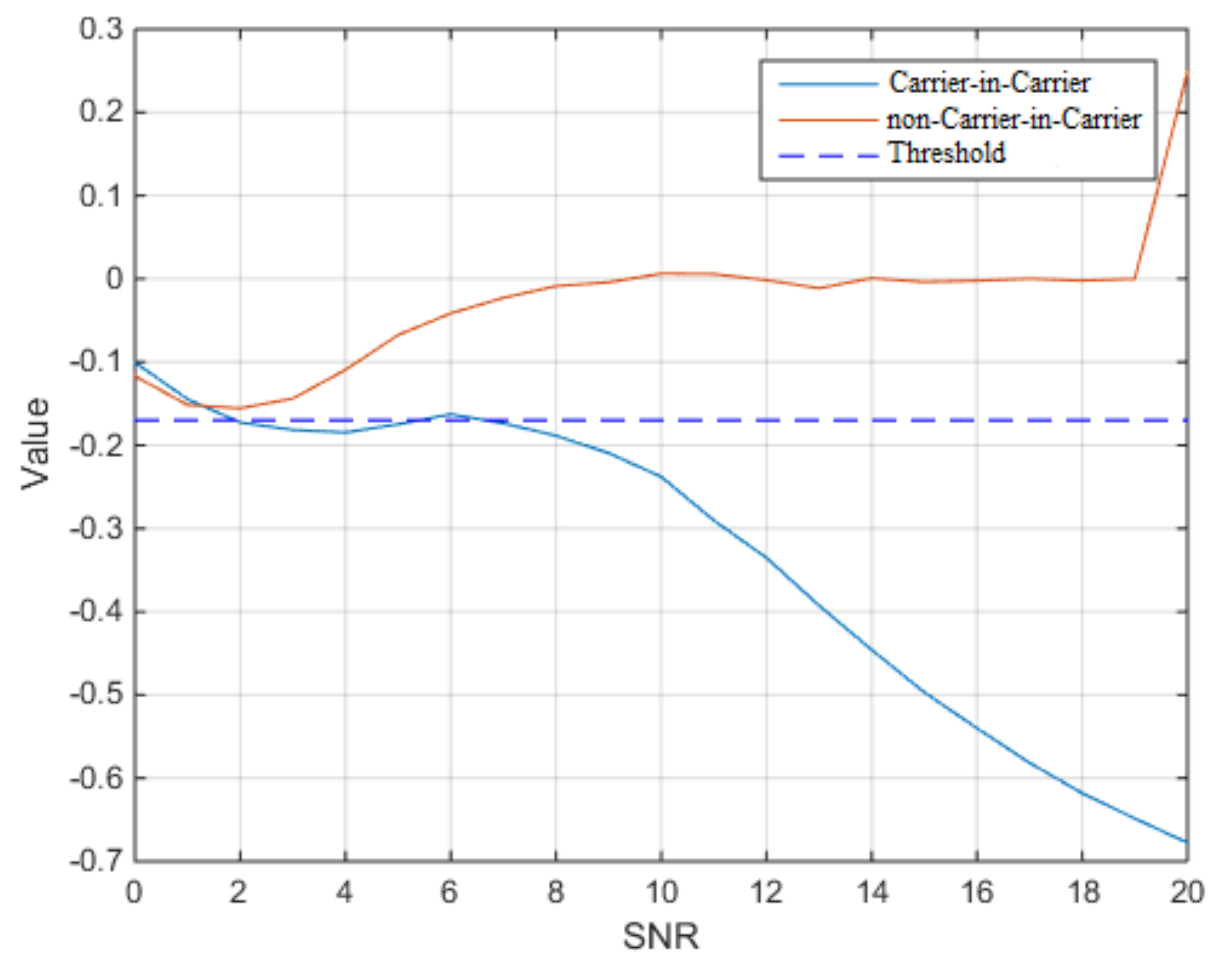

Figure 12: Dependence of average cumulants on the SNR for the case $T_{2} / T_{1}=0.25, a_{2} / a_{1}=2$.

The presented results show that the proposed detection rule suits equally well also for the case of different symbol rates.

\section{CONCLUSIONS}

In this paper, a method for the detection of the Carrier-in-Carrier signals based on the calculation of fourth-order signal cumulants was proposed. In accordance with the methodology based on the "Receiving operating curve" (ROC) parameter, a threshold value for the decision rule was established. It was found that the proposed method provides the correct detection of the sum of QPSK signals with SNR greater than $1.5 \mathrm{~dB}$, which corresponds to a wide class of practically encountered situations. The obtained AUC value for the cumulants method was 0.963, which indicates the high efficiency of the proposed detection method. The advantage of the proposed detection method over the "radiuses" method was also shown.

\section{REFERENCES}

[1] Agne, Craig \& Cornell, Billy \& Dale, Mark \& Keams, Ronald \& Lee, Frank, (2010) "Sharedspectrum bandwidth efficient satellite communications", Proceedings of the IEEE Military Communications Conference (MILCOM' 10), pp341-346.

[2] Gouldieff, Vincent \& Palicot, Jacques, (2015) "MISO Estimation of Asynchronously Mixed BPSK Sources", Proc. IEEE Conf. EUSIPCO, pp369-373.

[3] Semenov, Vasyl, (2018) "Method of Iterative Single-Channel Blind Separation for QPSK Signals", Mathematical and computer modelling, Vol. 17, No. 2, pp108-116. 
[4] Feng, Hao \& Gao, Yong, (2016) "High-Speed Parallel Particle Filter for PCMA Signal Blind Separation”, Radioelectronics and Communications Systems, Vol.59, No.10, pp305-313.

[5] Meyer-Basea, Anke \& Grubera Peter \& Theisa, Fabian,and Foo, Simon, (2006) "Blind source separation based on self-organizing neural network", Eng. Appl. Artificial Intelligence, Vol. 19, pp305-311.

[6] Fernandes, Carlos Estevao R. \& Comon, Pierre \& Favier, Gerard, (2010) "Blind identification of MISO-FIR channels", Signal Processing, Vol. 90, pp490-503.

[7] Swami, Anantharam \& and Sadler, Brain M., (2000) "Hierarchical digital modulation classification using cumulants,” IEEE Trans. Commun., Vol. 48, pp416-429.

[8] Wunderlich, Adam \& Goossens, Bart \& Abbey, Craig K. "Optimal Joint Detection and Estimation That Maximizes ROC-Type Curves” (2016) IEEE Transactions on Medical Imaging, Vol. 35, No.9, pp2164- 2173.

\section{AUTHORS}

Vasyl Semenov received a Ph.D. in Acoustics from Institute of Hydromechanics of National Academy of Sciences of Ukraine in 2004. He is currently the chief of the Department of Algorithms at Delta SPE LLC, Kiev, Ukraine and doctoral student at the Institute of Cybernetics of National Academy of Sciences of Ukraine. His main research interests are in the fields of digital signal processing, demodulation, blind separation, and recognition systems.

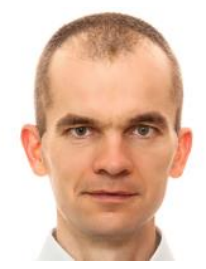

Pavel Omelchenko received a Ph.D. in Mathematics from Institute of Mathematics of National Academy of Sciences of Ukraine in 2010. He is currently the member of the Department of Algorithms at Delta SPE LLC, Kiev, Ukraine. His main research interests are in the fields of digital signal processing, demodulation, blind separation, and cryptanalysis systems.

Oleh Kruhlyk received M.Sc. degree in Radioelectronics from the National Technical University of Ukraine "Kiev Polytechnic Institute" in 2017. He is currently the member of the Department of Algorithms at Delta SPE LLC, Kiev, Ukraine and Ph.D. student at the National Technical University of Ukraine "Kiev Polytechnic Institute". His main research interests are in the fields of digital signal processing, demodulation, and blind separation and methods. 